\title{
Outbreak of acute hepatitis B virus infection associated with exposure to acupuncture
}

\author{
Rempel $\mathrm{S}^{1,2^{\star}}$, Murti $\mathrm{M}^{1}$, Buxton $\mathrm{JA}^{3}$, Stephens $\mathrm{W}^{4}$, Watterson $\mathrm{M}^{4}$, Andonov $\mathrm{A}^{2}$, Fung $\mathrm{C}^{1}$, Ramler $\mathrm{G}^{1}$, \\ Bigham $\mathrm{M}^{1}$, Lem $\mathrm{M}^{1}$
}

\section{Abstract}

Background: The most common risk factors for acute hepatitis B virus (HBV) infection are sexual contact, injection drug use and perinatal, or nosocomial exposure. Acupuncture, used in China for over 2,500 years, has been gaining popularity as an alternative medical therapy in the western world, but when associated with poor infection control practices, is also a risk for blood-borne infections.

Objective: To describe the outbreak investigation following detection of two cases of acute HBV infection associated with acupuncture services from the same provider within four months of symptom onset.

Methods: The outbreak investigation included genotyping of HBV from the identified cases, on-site assessment of the acupuncturist's infection prevention and control practices and chart review of known clients.

Results: Both cases had HBV genotype D1 with an identical fingerprint and both clients had visited the clinic on the same day denying other recent risk exposures. Inspection of the acupuncturist's practice revealed high-risk re-use and inappropriate storage of disposable needles. The Regional Health Authority ordered cessation of clinic practice until infection control measures were remediated. A public service announcement and mailed notifications to clients identified from practitioner records recommended that all clients be tested for HBV, human immunodeficiency virus (HIV) and hepatitis C.

Conclusions: A clear epidemiological linkage of these two acute HBV infections to the same acupuncture clinic, evidence of substandard infection control practice in the clinic and identical HBV molecular and genotypic profiles of the two cases are highly suggestive that contaminated acupuncture needles likely resulted in at least two cases of acute HBV infection. This is the first known reported transmission of HBV from acupuncturists re-use of disposable needles and the first HBV outbreak associated with exposure to acupuncture reported this century in an industrialized country. Increased provider oversight and patient education may prevent future outbreaks.

\author{
Affiliations \\ ${ }^{1}$ Fraser Health Authority, Surrey, \\ $\mathrm{BC}$ \\ ${ }^{2}$ Public Health Agency of Canada, \\ Ottawa, ON \\ ${ }^{3}$ British Columbia Centre for \\ Disease Control, Vancouver, BC \\ ${ }^{4}$ College of Traditional Chinese \\ Medicine Practitioners and \\ Acupuncturists of British \\ Columbia, Vancouver, BC
} *Correspondence: shirley.
rempel@gmail.com

Suggested citation: Rempel S, Murti M, Buxton JA, Stephens W, Watterson M, Andonov A, et al. Outbreak of acute hepatitis B virus infection associated with exposure to acupuncture. Can Comm Dis Rep 2016;42:169-72. https://doi.org/10.14745/ccdr.v42i08a04

\section{Introduction}

Two cases of acute hepatitis B virus (HBV) infection were reported to a Regional Health Authority in British Columbia (BC) between July and October, 2014. The previous five-year annual (2009-2013) average of reported acute HBV for this region was 1.2 cases. Case A, a 54 year-old male, was hospitalized for symptoms of acute hepatitis (jaundice, dark urine, right upper quadrant abdominal pain, nausea, malaise, fatigue and loss of appetite) in July, 2014. Case B, a 53 year-old female, visited the hospital emergency room in October 2014, 13 days after onset of a similar clinical illness. Both were diagnosed with acute HBV infection based on clinical history and serology. Enhanced Hepatitis Strain Surveillance System (EHSSS) case reports $(1,2)$ identified acupuncture as the only significant risk factor for both clients, who notably recalled receiving services on the same day from a traditional Chinese medicine acupuncture clinic (Clinic A) within four months of symptom onset.

Data on hepatitis C virus (HCV) infection in Canada is based on mandatory reporting of cases to the Canadian Notifiable Disease Surveillance System (CNDSS) by provincial and territorial health authorities. However, CNDSS has a limited ability to systematically detect incident infections. Therefore enhanced hepatitis strain surveillance involving the collection of additional epidemiological and laboratory information was undertaken to improve the yield of incident cases $(2,3)$. BC continued using the EHSSS HBV case reporting forms after the EHSSS was 
discontinued in 2012 (1). An astute public health nurse identified the common exposure while interviewing the second case. Based on these findings a HBV outbreak was declared and an investigation initiated.

Acupuncture, used in China for over 2,500 years, has been gaining popularity as an alternative medical therapy in the western world over the past two decades (4). A review of English-language publications found six HBV outbreaks associated with acupuncture reported between 1977 and 1999 (5). Most HBV outbreaks were due to patient-to-patient transmission and only one outbreak was reported from provider-to-patient transmission $(6,7)$. HBV infection is a rare complication in industrialized countries where licensure of acupuncturists is generally required.

$B C$ rates of acute HBV have been less than one per 100,000 population since 2007 and lower than the national average since universal childhood HBV vaccine was introduced. Cases occur in adults 25 years of age and older and more frequently in males than females (8-10). HBV is transmitted through exposure to infectious blood and body fluids, most commonly acquired via sexual contact, injection drug use, perinatal, or nosocomial exposure (8). The incubation period usually ranges from 45-180 days with an average of 60-90 days (10). Acute infection can be severe, resulting in acute hepatic necrosis, however the majority of adult cases (50-70\%) are asymptomatic and remain undetected. The case-fatality-rate is approximately one percent. The risk of developing chronic infection is highest (20-50\%) in children five years and younger, decreasing to one to ten percent in older age groups $(8,11)$.

The objective of this report is to describe the epidemiological, genetic and public health investigation of this outbreak.

\section{Methods}

The outbreak investigation was designed to assess the hypothesis that acupuncture was the probable source of transmission, identify those at risk, undertake active case-finding and prevent further spread. The investigation included genotyping and molecular fingerprinting of HBV isolates from the identified cases, a chart review of Clinic A clients, on-site inspection of the practitioner's infection prevention and control practices and review of acute HBV and HCV cases reported in the region over the previous 10 years.

There was insufficient information to determine a risk period for potential practice-associated nosocomial blood-borne infection at Clinic $A$; therefore, the time frame chosen for potential cases extended back to 2004 when the clinic opened, until it closed in 2014.

The following case definition, adapted from BC provincial guidelines was used for this outbreak: Hepatitis B surface antigen ( $\mathrm{HBsAg}$ ) and immunoglobulin $\mathrm{M}$ antibody to hepatitis $B$ core antigen (anti-HBc lgM) positive in the context of a compatible clinical history (8) and epidemiologic link to Clinic A between January 2004 and November 14, 2014.

\section{Results}

A review of acute HBV and HCV cases reported in the Regional Health Authority over the past 10 years was conducted to determine if there were any additional cases linked to Clinic A. The majority of cases were diagnosed prior to the establishment of the EHSSS and as such, efforts were made to contact cases by telephone to determine if they had been exposed to acupuncture. Interviews of acute HBV cases and reviews of acute $\mathrm{HBV}$ and $\mathrm{HCV}$ cases reported in the region did not identify additional acute hepatitis cases linked to Clinic $A$.

The two cases were in a male and a female, 54 and 53 years of age respectively, both residing in the Regional Health Authority. The only HBV risk exposure that both cases reported was receiving acupuncture at the same traditional Chinese medicine clinic. Both known cases attended this clinic between May and June 2014 and reported receiving acupuncture on the same day in May. Phylogenetic analysis by the National Microbiology Laboratory revealed that both cases had HBV genotype D1 with an identical S-gene "fingerprint" which was highly suggestive of a common source of infection. The acupuncture practitioner had no serologic evidence of prior or current HBV infection.

Clinic A's chart documentation was limited to the first appointment, with no subsequent documentation in patient charts of dates for follow-up appointments. Treatment notes were rare and all had dates missing.

A joint inspection of the acupuncture clinic conducted by a Regional Health Authority Medical Health Officer and the College of Traditional Chinese Medicine Practitioners and Acupuncturists of BC Registrar (the provincial regulator) revealed unhygienic conditions and poor infection control practices. Treatment rooms lacked sinks with hot and cold running water and easily cleanable surfaces. Standardized procedures for disinfection, hand washing and separation of clean and dirty fields were not practiced. Of greatest significance was the finding of single-use acupuncture needles stored in open bowls, open needle packaging and absence of sharps disposal containers, suggesting a high probability of needle re-use.

Given the risks identified during the inspection, the College of Traditional Chinese Medicine Practitioners and Acupuncturists provided notice of immediate temporary license suspension on November 14, 2014 and the Regional Health Authority ordered the practitioner to cease practice on November 15, 2014. There was insufficient information to determine the duration of these unsafe practices given the absence of a previous inspection or monitoring, which led to the determination that all patients of Clinic A were potentially at risk of nosocomial exposure to blood-borne pathogens.

Notifications were mailed to 1,374 of the 1,516 clients identified from clinic intake forms who had valid address information. An estimated 1,200 (79\%) of clients received notifications (174 notifications were returned as non-deliverable). A public service announcement was issued by the Regional Health Authority on November 20, 2014, recommending that all clients who had ever received services from Clinic A be tested for HBV, HIV and HCV. Anticipating public concern, a call centre staffed 
by Regional Health Authority public health nurses was established. The centre received 114 calls: 103 were from concerned clients or their families and 87 of these individuals were referred to their family doctor for testing. The total number of clients who went for testing is unknown because negative infectious disease test results are not reported to public health in BC.

Once general hygiene and infection control measures were remediated, the College of Traditional Chinese Medicine Practitioners and Acupuncturists lifted the registrant's practice suspension and the Regional Health Authority amended the Order on November 26, 2014, permitting conditional resumption of clinical services at Clinic $A$, subject to satisfactory findings in ongoing inspections and monitoring.

\section{Discussion}

Contaminated acupuncture needles were identified as the likely cause of two cases of acute HBV infection, based on the epidemiologic evidence and identical HBV molecular and genotypic profiles. To our knowledge, this is the first known reported transmission of HBV from acupuncturists re-use of disposable needles and the first HBV outbreak associated with exposure to acupuncture reported this century in an industrialized country.

Access to phylogenetic analysis was a key strength of this investigation as it provided strong evidence of a common source of infection. The investigation was limited by its inability to determine a denominator of the number of acupuncture clinic clients who underwent the recommended testing.

$\mathrm{HBV}$ infection is vaccine-preventable. Routine HBV immunization programs in $\mathrm{BC}$ have targeted infants and children and have resulted in the elimination of acute HBV within the immunized age cohort (12). Recommendations for adult HBV immunization are based on acquisition risk factors, such as occupation or close contact with a case of acute or chronic $\operatorname{HBV}(8,13)$. As such, the majority of adults born prior to 1982 remain susceptible to infection with HBV. More recently, receiving acupuncture treatment from a licensed provider is not generally considered a risk, based in large part on the expectation that routine infection control precautions and practices are in place and consistently followed $(14,15)$.

Publications of previous acupuncture-associated HBV outbreaks recommended prevention through enhanced provider regulations, provider training and sterilization or use of single-use needles as methods to eliminate the potential of future outbreaks (16-18).

Most industrialized countries regulate acupuncturists' practice directly with health practitioner legislation or indirectly by broad legislation governing the use of needles and other "sharps". In North America, related legislation is enacted at the state or provincial level $(19,20)$. The College of Traditional Chinese Medicine Practitioners and Acupuncturists was established in 1996 under the BC Health Professions Act to regulate the practice of traditional Chinese medicine and acupuncture. Other health professions may provide acupuncture in accordance with requirements of their regulating bodies. Annual practitioner registration under the provincial Health Professions Act requires attestation that by-laws related to such matters as infection control practices are being followed. Compliance inspection or monitoring is not routinely undertaken by the College of Traditional Chinese Medicine Practitioners and Acupuncturists unless it becomes evident that a registrant poses a public health risk (19). The acupuncturist in this instance was licensed with the College of Traditional Chinese Medicine Practitioners and Acupuncturists as a registrant in good standing, but failed to comply with practice standards and ongoing education requirements.

Licensing requirements of North American traditional Chinese or Oriental medicine colleges include satisfactory completion of training at an accredited institute $(19,20)$. Infection control practices to prevent transmission of blood-borne pathogens through the use of needles are a fundamental component of training and certification examinations for all regulated health professionals. A multi-media public information campaign launched by the BC Health Regulators in September 2014 used transit shelter advertisements, articles in community papers, television closed-captioning messages and a new website which emphasized the importance of consulting licensed health professionals in good standing with their regulating body $(15,21)$. Proactive public education could also promote patient awareness of the importance of appropriate use and disposal of single-use needles and other infection prevention and control measures by health professionals during health care encounters.

Acupuncture, when performed in accordance with proper infection control procedures, is safe (14). Exposure to HBV is a risk formerly associated with the improper sterilization of reusable needles $(16,17)$. Single-use disposable needles are the current practice standard in the majority of industrialized countries (22). The clean needle technique is a term used by licensing agencies to define required equipment and processes to provide acupuncture. Developed specifically to prevent transmission of HBV by acupuncturists, clean needle technique requires the use of sterile, single-use needles for acupuncture and confirmation that each needle used has been discarded in an appropriate disposal container (22).

\section{Conclusion}

Based on the epidemiologic investigation and identical molecular HBV genotypic profiles, re-use of contaminated acupuncture needles intended for single-use likely resulted in at least two cases of acute HBV infection. The incubation period for HBV usually ranges from 45-180 days (11) with an average of 60-90 days. Exposure ended on November 14, 2014, however given that adults may develop subclinical chronic HBV infection (11), identification of future cases associated with this outbreak remains possible.

To our knowledge, this is the first HBV outbreak associated with exposure to acupuncture reported this century in an industrialized country despite existing regulations regarding safe infection control practices, requirements for extensive provider training and use of disposable needles. The coordinated response between the Regional Health Authority and the College of Traditional Chinese Medicine Practitioners and Acupuncturists ended the potential for transmission of HBV and other blood-borne pathogens from that clinic. Ongoing 
vigilance by public health and the College of Traditional Chinese Medicine, ensuring providers are meeting practice standards and actively educating consumers are critical to preventing future outbreaks.

\section{Acknowledgements}

We thank the Fraser Health Communicable Disease Nurse Coordinator, the public health nurses and environmental health officer who staffed the call centre during the outbreak, conducted individual case investigations and follow-up and inspected Clinic A. Thank you to Gail Kyle who noted the common exposure that expedited detection of the outbreak. We also thank the BC Centre for Disease Control Public Health and Microbiology Reference and Microbiology Laboratory.

\section{Conflict of interest}

None.

\section{References}

1. $B C$ Centre for Disease Control (BCCDC). Administrative circular 04: New case report forms for acute hepatitis B and acute hepatitis C. Vancouver: BCCDC; March 2016. http:// www.bccdc.ca/resource-gallery/Documents/Guidelines\%20 and\%20Forms/Guidelines\%20and\%20Manuals/Epid/CD\%20 Manual/Admin\%20Circulars/2016/AC_2016_04_Acute_ HepatitisBC.pdf.

2. Wu H-X, Wu J, Wong T, Donaldson T, Dinner K, Andonov A, et al. Enhanced surveillance of newly acquired hepatitis $C$ virus infection in Canada, 1998 to 2004 . Scand J Infect Dis 2006;38(6-7):482-9.

3. Public Health Agency of Canada (PHAC). Canadian Immunization Guide: Part 4, Active vaccines. Ottawa: PHAC; c2004-2016. http://www.phac-aspc.gc.ca/publicat/cig-gci/p04hepb-eng.php\#a1.

4. World Health Organization (WHO). Guidelines on basic training and safety in acupuncture. Geneva: WHO; The Organization; c1948 - 2015. 1999. http://apps.who.int/iris/ bitstream/10665/66007/1/WHO_EDM_TRM_99.1.pdf.

5. Woo PCY, Lin AWC, Lau SKP, Yuen K-Y. Acupuncture transmitted infections. BMJ 2010 Mar 18;340(mar18 1):c1268c1268.

6. Walsh B, Maguire H, Carrington D. Outbreak of hepatitis B in an acupuncture clinic. Commun Dis Public Health PHLS 1999 Jun;2(2):137-40.

7. Slater PE, Ben-Ishai P, Leventhal A, Zahger D, Bashary A, Moses A, et al. An acupuncture-associated outbreak of hepatitis B in Jerusalem. Eur J Epidemiol 1988 Sep;4(3):322-5.

8. BC Centre for Disease Control (BCCDC). Communicable disease control. Chapter 1: Management of specific diseases, hepatitis B. Vancouver: BCCDC; c1998-2015 [updated 2009 September]. http://www.bccdc.ca/resource-gallery/
Documents/Guidelines\%20and\%20Forms/Guidelines\%20 and\%20Manuals/Epid/CD\%20Manual/Chapter\%201\%20-\%20 CDC/HepatitisB_Sept_2009.pdf.

9. Public Health Agency of Canada (PHAC). Hepatitis B infection in Canada: Brief report. Ottawa: PHAC; c2004-2016 [updated 2011]. http://www.phac-aspc.gc.ca/id-mi/hepatitisBCanhepatiteBCan-eng.php.

10. Public Health Agency of Canada (PHAC) Report on plans and priorities 2015 - 2016. Ottawa: PHAC; c2004-2016 [updated 2016]. http://www.phac-aspc.gc.ca/rpp/2015-2016/assets/pdf/ rpp-2015-2016-eng.pdf.

11. Heymann DL, American Public Health Association. Control of communicable diseases manual. Washington, DC: American Public Health Association; 2008.

12. Patrick DM, Bigham $\mathrm{M}, \mathrm{Ng} \mathrm{H}$, White $\mathrm{R}$, Tweed A, Skowronski DM. Elimination of acute hepatitis $B$ among adolescents after one decade of an immunization program targeting Grade 6 students. Pediatr Infect Dis J 2003 Oct;22(10):874-7.

13. Centers for Disease Control and Prevention (CDC) Immunology and vaccine-preventable diseases: Pink book: Hepatitis B. Atlanta GA: CDC; c1946-2015 [updated 2015 Apr]. http://www.cdc.gov/vaccines/pubs/pinkbook/ downloads/hepb.pdf.

14. Government of British Columbia HealthLink BC. Acupuncture topic overview. Vancouver: Healthwise BC; c1995-2015 [updated 2015 July 15]. http://www.healthlinkbc.ca/ healthtopics/content.asp?hwid=aa77639spec.

15. BC Health Regulators website. Vancouver: BC Health Regulators; c2014-2016 [updated 2014]. http://www. bchealthregulators.ca/.

16. Stryker WS, Gunn RA, Francis DP. Outbreak of hepatitis B associated with acupuncture. J Fam Pract 1986 Feb;22 (2):155-8.

17. Boxall EH. Acupuncture hepatitis in the West Midlands, 1977. J Med Virol 1978;2(4):377-9.

18. Kent GP, Brondum J, Keenlyside RA, LaFazia LM, Scott HD. A large outbreak of acupuncture-associated hepatitis B. Am J Epidemiol 1988 Mar;127(3):591-8.

19. College of Traditional Chinese Medicine Practitioners and Acupuncturists. Bylaws. Vancouver: The College; c19992015 [updated 2015 Apr 1]. http://ctcma.bc.ca/about/ announcements/2016-06-18-resolution-of-the-board-ofctcma/.

20. National Certification Commission for Acupuncture and Oriental Medicine. NCCAOM State licensure requirements. Jacksonville; NCCAOM; c1982-2015. http://www.nccaom.org/ regulatory-affairs/state-licensure-map.

21. College of Massage Therapists of British Columbia. BC Health Regulators launch public safety campaign: "Saying you are one doesn't make you one". The College; c1994-2016 [updated 2015 Nov]. http://www.cmtbc.ca/news/2015/10/14/ bc-health-regulators-launch-public-safety-campaign\%E2\%80\%9Csaying-you-are-one-doesn\%E2\%80\%99t-make.

22. Council of Colleges of Acupuncture and Oriental Medicine. Clean needle technique manual. Baltimore: The Council; c1982-2015 [updated 2015 Sep]. http://www.ccaom.org/down loads/7thEditionManualEnglishPDFVersion.pdf. 